\title{
Design Rules for Single-Mode and Polarization- Independent Silicon-on-Insulator Rib Waveguides Using Stress Engineering
}

\author{
Milan M. Milošević, Petar S. Matavulj, Member, IEEE, Branislav D. Timotijević, Graham T. Reed, and \\ Goran Z. Mashanovich
}

\begin{abstract}
There is a trend towards miniaturization of silicon photonic circuits due to superior performance and small cost. Design rules that must be imposed on the geometry of optical waveguides to make them behave as polarization-independent and singlemode devices are well known for waveguides with relatively large cross sections and for some small cross-sectional rib waveguides with vertical sidewalls and an air top cladding. The influence of the top oxide cover on waveguide birefringence was analyzed recently, but only for relatively large cross-sectional waveguides. This paper reports simulations for both single-mode and polarization-independent behavior for small cross-sectional waveguides with variable rib width, etch depth, top oxide cover thickness, and sidewall angle. The results show that the stress-induced effects must be taken into account to satisfy both requirements. Design rules to maintain birefringence-free operation and to satisfy single-mode behavior for small rib silicon-on-insulator (SOI) waveguides are presented.
\end{abstract}

Index Terms-Birefringence, polarization independence, rib waveguides, silicon photonics, single mode, stress.

\section{INTRODUCTION}

$\mathbf{R}$ ECENT years have seen an enormous interest in increased functionality and cost-effective fabrication of smaller silicon photonic devices. An optical waveguide, built in the most promising silicon-on-insulator (SOI) platform, represents the basic building component in many optical systems, such as arrayed waveguide gratings (AWGs), resonators, modulators, lasers, and tunable systems. High-index contrast between the cladding and waveguide core facilitates the optical mode confinement and guiding of light. Typical wavelengths for SOI applications are 1.31 and $1.55 \mu \mathrm{m}$, but longer wavelengths are not suitable (except in the $3-3.5-\mu \mathrm{m}$ range) due to absorption spectra of silicon dioxide [1]. Integration with complimentary

Manuscript received December 28, 2007; revised February 26, 2008. Published August 29, 2008 (projected). This work was supported in part by the U.K. Royal Society International Joint Project under Contract 2006/R2-IJP and in part by the Serbian Ministry of Science under Contract 141006.

M. M. Milošević and P. S. Matavulj are with the Faculty of Electrical Engineering, University of Belgrade, Belgrade 11120, Serbia (e-mail: milance24@gmail.com; matavulj@etf.bg.ac.yu; p.matavulj@ieee.org).

B. D. Timotijević is with the VINČA" Institute of Nuclear Sciences, Belgrade 11001, Serbia (e-mail: bane@vin.bg.ac.yu).

G. T. Reed and G. Z. Mashanovich are with the Advanced Technology Institute, University of Surrey, Guildford GU2 7XH, U.K. (e-mail: g.reed@surrey.ac.uk; g.mashanovich@surrey.ac.uk).

Color versions of one or more of the figures in this paper are available online at http://ieeexplore.ieee.org.

Digital Object Identifier 10.1109/JLT.2008.922193 metal-oxide-semiconductor (CMOS) [2], Raman silicon lasers [3], 30-Gb/s modulators [4], compact wavelength routers [5], low-loss waveguides [6], [7], and all-optical switching [8] represent valuable applications that have strengthened the position of silicon photonics and utilization of the SOI platform.

Unlike silica waveguides that can be relatively easily designed to be single mode, SOI waveguides with dimensions larger than a few hundred nanometers in cross section will potentially support multiple modes. Such waveguides are usually undesirable in photonic circuits as their operation can be seriously compromised by the presence of multiple modes. It has been shown, however, that large rib waveguides in SOI could be designed to be monomodal [9]. These waveguides have been studied extensively by a number of researchers [10]-[13] to find single-mode behavior and low-loss propagation. Large rib waveguides are interesting because they are multimicron in cross-sectional dimensions (of the order of $5 \mu \mathrm{m}$ ) facilitating low-loss coupling to and from optical fibres. Soref et al. [9] first proposed a simple expression for the single-mode condition (SMC) of such waveguides

$$
\frac{W}{H} \leq 0.3+\frac{r}{\sqrt{1-r^{2}}}, \quad \text { for } 0.5 \leq r \leq 1.0
$$

where $r=h / H$ is the ratio of slab height to overall rib height, and $W / H$ is the ratio of waveguide width to overall rib height (Fig. 1). Their analysis of the waveguides was limited to shallow etched ribs $(r>0.5)$ and the waveguide dimensions were assumed to be larger than the operating wavelength. The analysis was based on the assumption that higher order vertical modes (i.e., modes other than the fundamental mode) confined under the rib, were coupled to the outer slab region during propagation, therefore yielding high propagation losses for the higher order modes. Thus, the waveguides behave as single-mode waveguides, as all other modes are lost. Chan et al. [14] produced equations to predict single mode and polarization independence for relatively small rib waveguides

$$
\begin{aligned}
\frac{W}{H} \leq & 0.05+\frac{(0.94+0.25 H) r}{\sqrt{1-r^{2}}}, \\
& \quad \text { for } 0 \leq r \leq 0.5 \text { and } 1.0 \mu \mathrm{m} \leq H \leq 1.5 \mu \mathrm{m} \\
D_{\min }= & 0.06 \times 10^{-6}+0.556 H .
\end{aligned}
$$

Equation (2) defines the quasi-TM (transverse magnetic) singlemode boundary, and hence provides guidance on the geometrical limitations to retain single-mode behavior, while (3) defines the minimum etch depth $D_{\min }$ required to obtain polariza- 


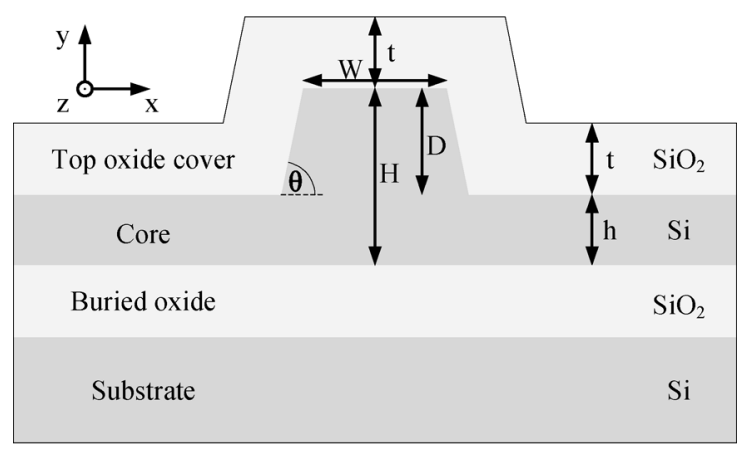

Fig. 1. Cross section of SOI rib waveguide: $t$ is the top oxide layer thickness, $H$ is rib height, $D(D=H-h)$ is etch depth, $W$ is waveguide rib width, and $\theta$ is the rib sidewall angle.

tion independence. The guidelines of (2) and (3) are for waveguides with an air top cladding. However, an upper cladding layer is often deposited to reduce the influence of surface contamination, to passivate the surface, or to provide electrical isolation. For the SOI platform, this layer is usually $\mathrm{SiO}_{2}$ (Fig. 1), although nitride and polymer layers are also used in some cases. Therefore, the single-mode and birefringence-free conditions also need to be determined for such a cladding, not only because oxide cladding has a different refractive index than air, but also because it causes stress in the waveguide structure therefore changing effective refractive indexes for (transverse electric) TE and TM polarizations. Recent simulations have shown that the single-mode/multimode boundary for TM polarization is below the TE boundary and the following equation for the multimode boundary was given [15]:

$$
W[\mu \mathrm{m}] \leq 2.86 \times(D[\mu \mathrm{m}])^{2}-7.42 \times D[\mu \mathrm{m}]+4.75 .
$$

This equation is more strict than (2), but it is based on an isotropic distribution of material refractive index and is valid only for waveguides with a large top oxide layer $(t \sim 1 \mu \mathrm{m})$ and vertical waveguide sidewall angle. In this paper, we analyze the difference between isotropic and anisotropic cases. The influence of a thinner oxide cover and waveguide sidewall angle is also discussed.

In many applications, it is important to achieve small modal birefringence, defined here as the difference between the effective indexes of the two orthogonally polarized modes, the horizontally polarized mode (quasi-TE) and the vertically polarized mode (quasi-TM) $\Delta N_{\text {eff }}=N_{\text {eff }}^{\mathrm{TE}}-N_{\text {eff }}^{\mathrm{TM}}$. To achieve zero birefringence (ZBR) in rib waveguides, an optimization of waveguide dimensions is necessary [16]-[18]. As we are investigating a most common situation where there is an oxide upper cladding, the total birefringence is, however, the sum of the geometrical birefringence and stress-induced birefringence [16]. The structure we analyze in this paper, at the operating wavelength of $\lambda=1.55 \mu \mathrm{m}$, is a rib waveguide with the height of $H=1.35 \mu \mathrm{m}$, and variable waveguide rib width $W$, etch depth $D$, top oxide cover thickness $t$, and sidewall angle $\theta$, as shown in Fig. 1. The buried oxide (BOX) layer is $1 \mu \mathrm{m}$ in thickness, while the top oxide cladding in performed simulations is $0.1-3$ $\mu \mathrm{m}$ thick. These waveguide dimensions are chosen as we have previously reported optical filters based on such waveguide [19],
[20]. The strain in the upper $\mathrm{SiO}_{2}$ layer produces a stress distribution within and near the Si rib, which in turn causes a change of the refractive index in both materials due to the photoelastic effect. During the simulations, we used the fact that the thickness of the upper cladding film on the rib sidewalls is $70 \%$ of that on the top, a result obtained by a scanning electron microscope (SEM) investigation of the fabricated rib waveguides. In this paper, we present the effect of stress on the multimode boundary and the effect of stress on the polarization independence curve. The simulations were performed by using a 2-D finite element method (FEM) modeling [21]-[23] and verified by 3-D semiand full-vectorial beam propagation method (BPM) [24]. The method we used was to calculate the effective indexes for the fundamental and the first two higher order modes, for both polarizations, over a range of waveguide dimensions.

This paper is organized as follows. In Section II, the stress theory and the stress influence on the total birefringence are presented. The polarization-independent surface and the influence of the waveguide sidewall angle is also explained. The singlemode behavior with and without taking into account stress-induced effects is given in Section III. The concluding remarks are summarized in Section IV.

\section{WAVEGUide StRESS THEORY AND POLARIZATION-INDEPENDENT SURFACE}

The stress causes birefringence [25], which may in turn induce polarization conversion and polarization-dependent loss. The change in the material effective indexes caused by stress-induced effects can be expressed as [26]-[30]

$$
\begin{aligned}
& \Delta N_{x}=N_{x}-N_{0}=-C_{1} \sigma_{x}-C_{2}\left(\sigma_{y}+\sigma_{z}\right) \\
& \Delta N_{y}=N_{y}-N_{0}=-C_{1} \sigma_{y}-C_{2}\left(\sigma_{x}+\sigma_{z}\right)
\end{aligned}
$$

where $\sigma_{x}, \sigma_{y}$, and $\sigma_{z}$ are the principal components of the relative stress tensor, $N_{x}$ and $N_{y}$ are the components of the material's refractive index, $N_{0}$ is the refractive index without stress, and $C_{1}$ and $C_{2}$ are the stress-optic constants related to the Young's modulus $(E)$, Poisson's ratio $(\nu)$, and the photoelastic tensor elements $\left(p_{11}\right.$ and $\left.p_{12}\right)$ as

$$
\begin{aligned}
C_{1} & =\frac{N^{3}}{2 E}\left(p_{11}-2 \nu p_{12}\right) \\
C_{2} & =\frac{N^{3}}{2 E}\left(-\nu p_{11}+(1-\nu) p_{12}\right) .
\end{aligned}
$$

One source of birefringence is strain due to the temperature difference before and after fabrication processes. The strain and stress are related as follows [31], [32]:

$$
\left[\begin{array}{l}
\varepsilon_{x} \\
\varepsilon_{y} \\
\varepsilon_{z}
\end{array}\right]=\frac{1}{E}\left[\begin{array}{ccc}
1 & -\nu & -\nu \\
-\nu & 1 & -\nu \\
-\nu & -\nu & 1
\end{array}\right]\left[\begin{array}{l}
\sigma_{x} \\
\sigma_{y} \\
\sigma_{z}
\end{array}\right]+\left[\begin{array}{l}
\alpha \Delta T \\
\alpha \Delta T \\
\alpha \Delta T
\end{array}\right]
$$

where $\varepsilon_{x}, \varepsilon_{y}$, and $\varepsilon_{z}$ are the principal strain components along the $x, y$, and $z$ direction, respectively, $\alpha$ is the thermal expansion coefficient, and $\Delta T=T_{0}-T_{\text {ref }}$ represents the difference between the operating temperature $T_{0}$ and reference temperature $T_{\text {ref }}$. At the reference temperature, which corresponds to the deposition temperature for an idealized oxide film on a silicon system, there is no intrinsic stress in any layer and all the 
TABLE I

EXPERIMENTAL VAlues of MATERIAL PARAMETERS OF $\mathrm{Si}$ AND $\mathrm{SiO}_{2}$ USED IN THE CALCULATIONS FOR THE WAVELENGTH OF $1550 \mathrm{~nm}$. ALl VALUES ARE TAKen From [16]

\begin{tabular}{c||c||c}
\hline Parameter & $\mathrm{Si}$ & $\mathrm{SiO}_{2}$ \\
\hline \hline$N_{0}$ & 3.476 & 1.444 \\
$p_{11}$ & -0.101 & 0.16 \\
$p_{12}$ & 0.0094 & 0.27 \\
$E(\mathrm{GPa})$ & 130 & 76.7 \\
$\nu$ & 0.27 & 0.186 \\
$\alpha\left(10^{-6} K^{-1}\right)$ & 3.6 & 0.54 \\
\hline
\end{tabular}

material layers are at equilibrium. As it can be seen from (7) the strain consists of the photoelastic stress and thermally induced stress. By inverting (7), the relation for the stress distribution tensors can be obtained as

$$
\begin{array}{r}
{\left[\begin{array}{c}
\sigma_{x} \\
\sigma_{y} \\
\sigma_{z}
\end{array}\right]=\frac{E}{(1+\nu)(1-2 \nu)}\left[\begin{array}{ccc}
1-\nu & \nu & \nu \\
\nu & 1-\nu & \nu \\
\nu & \nu & 1-\nu
\end{array}\right]\left[\begin{array}{l}
\varepsilon_{x} \\
\varepsilon_{y} \\
\varepsilon_{z}
\end{array}\right]} \\
\quad-\frac{\alpha E \Delta T}{1-2 \nu}\left[\begin{array}{l}
1 \\
1 \\
1
\end{array}\right] .
\end{array}
$$

Table I summarizes all parameters used in numerical computations. Calculation window size was chosen to be large enough to minimize the influence of the edge effects on the stress distribution in the vicinity of the rib waveguide. Because of the sharp waveguide corners stress is inhomogeneous and anisotropic [17]. Due to the fabrication processes where the silicon core is covered by the silica cladding layer at high temperature, the difference between the operating $\left(20^{\circ} \mathrm{C}\right)$ and reference $\left(1000{ }^{\circ} \mathrm{C}\right)$ temperatures is assumed to be $\Delta T=-980 \mathrm{~K}$ [16]. The stress field in the waveguide and Maxwell's equations are solved by FEM, on a nonuniform mesh of triangular elements [21]. For our numerical computations, approximately 40000 elements and the higher order shape functions of the Lagrange type were used.

The modal birefringence was evaluated for different rib waveguide geometries and top oxide cover thicknesses in the $0.1-3-\mu \mathrm{m}$ range. The waveguide height was fixed at $H=$ $1.35 \mu \mathrm{m}$. First, we calculated the stress distribution and corresponding distribution of the refractive index in the waveguide. Then, Maxwell's equations were solved and effective indexes for both quasi-TE and quasi-TM modes were obtained from the eigenvalues [17], [18].

Fig. 2(a)-(c) represents the influence of waveguide rib width on the waveguide birefringence for a given value of the etch depth. We assumed vertical rib sidewalls and for a given top oxide thickness, for each etch depth, a series of $\Delta N_{\text {eff }}$ points were calculated. The results show that for smaller etch depths $(D<0.7 \mu \mathrm{m})$ the birefringence is always positive, i.e., quasi-TE and quasi-TM polarizations cannot be equalized by changing values of waveguide width and top oxide cover. Deeper etched devices, on the contrary, show a tendency for two specific waveguide widths for which the ZBR condition is fulfilled. This means that by using stress it is possible to produce birefringence-free waveguides for two different waveguide widths when a deeper etch depth is employed. The increase of the
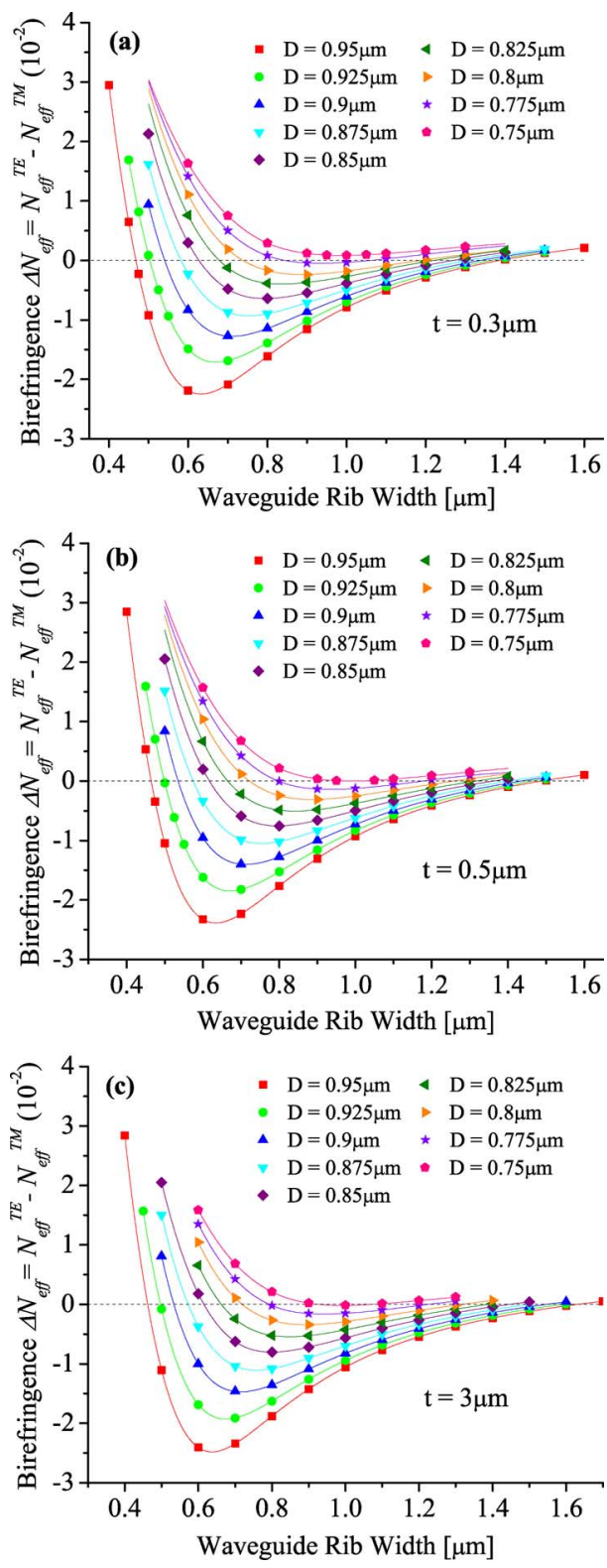

Fig. 2. Birefringence as a function of waveguide rib width and etch depth for top oxide thickness of (a) $t=0.3 \mu \mathrm{m}$, (b) $t=0.5 \mu \mathrm{m}$, and (c) $t=3 \mu \mathrm{m}$.

top oxide cover thickness will allow the presence of shallower etch depths at which the both modes will be guided through the waveguide in the same fashion. For larger $t$, the family of $\Delta N_{\text {eff }}$ curves becomes deeper and wider.

The ZBR curves for different values of top oxide cover thickness as a function of waveguide rib width and etch depth is presented in Fig. 3. It can be noticed that the increase of the top oxide cover thickness shifts the ZBR curves to smaller values of the etch depth, which is consistent with the results from Fig. 2. Also, considering constant etch depth, the zero-birefringence condition (ZBC) can be achieved for higher values of $W$, while the lower value of $D$ is "saturated." For the upper part of the ZBR curves, that is for wider ribs, when increasing the oxide thickness the birefringence will become negative and therefore to make it zero again, the width of the rib needs to be increased in order to reduce the stress influence on the rib. On the other hand, for the bottom part of the ZBR curves, that is for narrower 


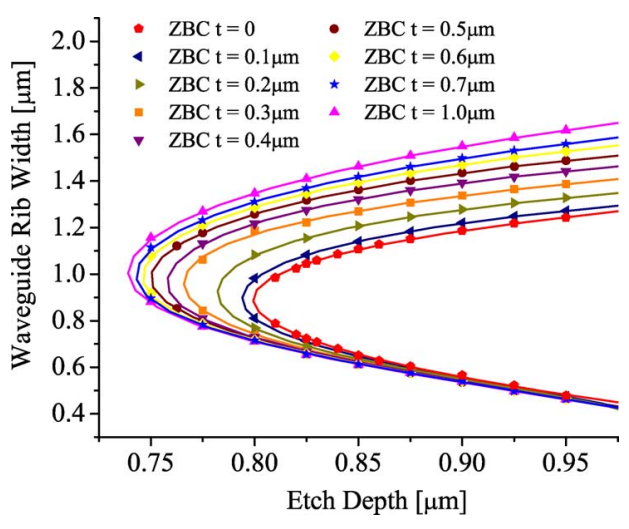

Fig. 3. ZBC as a function of waveguide rib width and etch depth for different values of top oxide thickness.

ribs, the mode resides mainly in the slab region and therefore the increase of the oxide thickness will not affect the birefringence significantly, hence the saturation in that region. The influence and importance of using stress engineering is obvious. The parabolas move to the left in respect to the $\mathrm{ZBC}$ curve for a rib waveguide with an air cover $(t=0)$. At large top oxide thicknesses $(t>0.8 \mu \mathrm{m})$ a saturation of ZBR curves is observed.

If we now calculate $Z B R$ points for each value of upper oxide thickness for each etch depth and corresponding waveguide rib width value, it is possible to draw the ZBR surface (Fig. 4). At the beginning $(t=0)$, the surface has parabolic shape, which becomes wider for larger values of $t$. Finally, it is saturated for $t>0.8 \mu \mathrm{m}$ and smaller $W$. We can observe that there is a minimum etch depth $\left(D_{\min }\right)$ for each waveguide design, which has to be matched if the ZBC needs to be met. The following equations describe the minimum etch depth for a given waveguide rib width and corresponding thickness of top oxide layer that have to be matched for polarization independent condition:

$$
\begin{aligned}
D_{\min }[\mu \mathrm{m}]= & 1.211-0.465 \times W[\mu \mathrm{m}], \\
& \text { for } 0.88 \mu \mathrm{m} \leq W \leq 1.03 \mu \mathrm{m} \\
t[\mu \mathrm{m}]= & 707.03 \times K^{3}-118.27 \times K^{2} \\
+ & 8.7826 \times K-0.0027
\end{aligned}
$$

where $K$ is connected with $D_{\min }$ in the following form:

$$
K=1.896-2.372 \times D_{\min }[\mu \mathrm{m}]
$$

It is interesting to see what happens to the ZBR curve when the sidewall angle is taken into account (Fig. 5). Here, four examples are presented, one with vertical rib sidewalls $\left(\theta=90^{\circ}\right)$ and three with the slanted sidewalls, $\theta \in\left(85^{\circ}, 80^{\circ}, 75^{\circ}\right)$, values that were measured on the fabricated rib waveguides [17], [33]. It can be seen that the ZBR curve shifts to lower values of $W$. When $\theta$ is decreased, for constant $W$ (width of the top of the rib), the width of the bottom of the rib will increase. In other words, the effective width of the rib will increase, and therefore for constant oxide thickness, the ZBC will not be fulfilled. To obtain $\Delta N=0$, the effective rib width need to be decreased, which means that the ZBC will be fulfilled for narrower ribs.

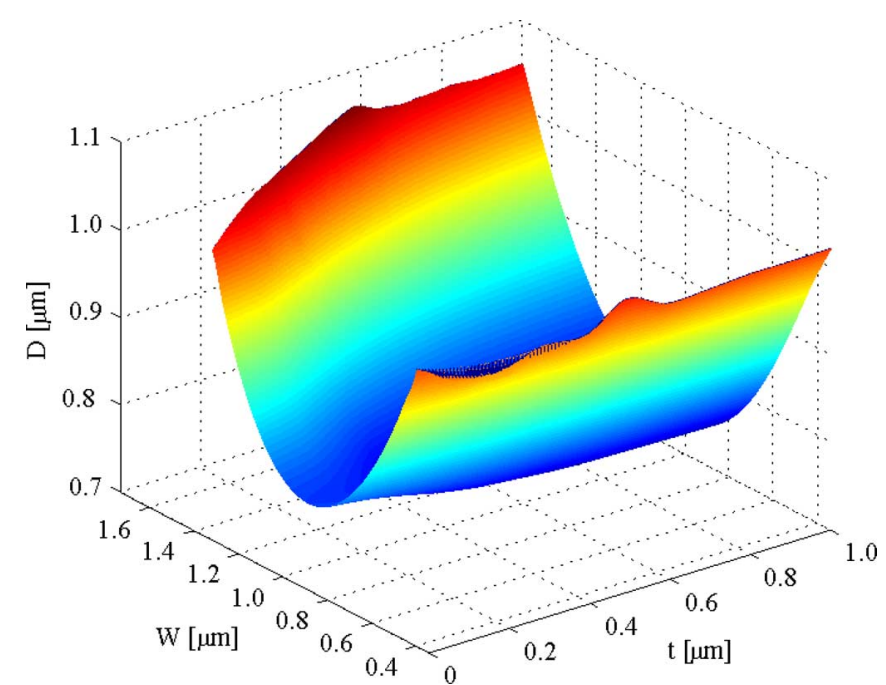

Fig. 4. ZBR surface for an operating wavelength of $\lambda=1.55 \mu \mathrm{m}$ and waveguide height of $H=1.35 \mu \mathrm{m}$.

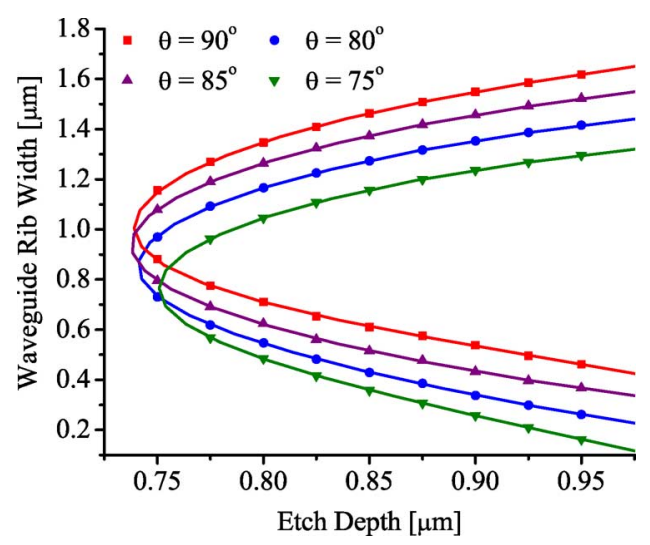

Fig. 5. ZBC as a function of waveguide rib width and etch depth for different values of ridge sidewall angle.

That is the reason why the ZBR curve shifts down in Fig. 5. When $\theta$ is decreased below $80^{\circ}$, the ZBR curve moves to the right, i.e., to larger $D$. We can conclude that the stress-induced birefringence for shallow etched rib waveguides has less influence on the total birefringence. Geometrical birefringence is then dominant and that is why the ZBR curve moves to the right, similar to the case when stress effects are not considered. Fabricated waveguides typically have sidewall angles in the $8^{\circ}-10^{\circ}$ range. In that case, the ZBR curve moves down maintaining the values of etch depth. If we assume this range of the sidewall angles, the following equation describes the influence of the sidewall to the waveguide width which maintains the ZBC:

$$
W[\mu \mathrm{m}]=-0.474+0.952 \times \theta[\mathrm{rad}], \text { for } 80^{\circ} \leq \theta \leq 90^{\circ} .
$$

This equation is complementary to the previous equations and they all together [(9)-(12)] give conditions for waveguide parameters that have to be matched to satisfy ZBC. As it can be seen, a careful design of waveguides need to be carried out prior to the fabrication to achieve polarization independence. 


\section{SATISFying Both Single-Mode AND} POLARIZATION-INDEPENDENCE CONDITIONS

The modal analysis of relatively small cross-sectional rib waveguides was performed by FEM simulations [21]-[23]. Maxwell's equations are solved in the two different cases, isotropic and anisotropic, due to stress-induced changes in material refractive indexes. The isotropic case is also solved via a full-vectorial BPM algorithm [24] to verify the FEM simulations. The incident field was chosen to be Gaussian beam lunched off-center with appropriate parameters that allowed excitation of higher order modes (modes other than the fundamental mode). To provide sufficient accuracy of FEM simulations with BPM, we used a correlation method [14] and calculated the fast Fourier transform (FFT) of the correlation function. This result can also be achieved by calculating the effective mode indexes using semivectorial BPM [33]. However, in this study, FEM simulations were used because they can easily incorporate the change in material refractive index caused by stress from the upper oxide layer via the photoelastic effect.

Similar to the calculation of the ZBR curves, for a given oxide thickness and sidewall angle, a number of single mode "quasi-TE" and "quasi-TM" points were found in the waveguide width/etch depth plane. We focused on one specific value for etch depth and changed the waveguide rib width until the first higher order mode was found. This was done for a given value of waveguide sidewall angle and top oxide layer thickness. Field profile is strongly dependent on waveguide geometry and in some cases we found that the more critical higher order mode was the quasi-TM $\mathrm{TM}_{01}$ mode, while in other cases quasi- $\mathrm{TM}_{10}$ mode was more critical. In common with our previous work (without stress-induced effects) [14], [33], we also found that satisfying the SMC for the TM mode is again more restrictive than for the TE mode. The area below the SMC for quasi-TM mode defines the global condition for the single-mode behavior. According to our simulations, for a given waveguide parameters, there is at least one intersection point between the SMC line for quasi-TM mode and the ZBR curve. A part of the ZBR parabola between these intersection points defines waveguide parameters for which both ZBC and SMC are satisfied.

Fig. 6 shows two different cases of the waveguide with vertical sidewalls. The first [Fig. 6(a)] is the waveguide without an upper oxide layer, while the second [Fig. 6(b)] represents the waveguide with an upper oxide cover of $t=0.3 \mu \mathrm{m}$ in thickness. In the first case [Fig. 6(a)], the same result as in [14] was obtained, as expected. Both the SMC and ZBC are satisfied and the quasi-TM curve is more critical for the single-mode/multimode boundary. In the case when an oxide layer is deposited, the situation is different [Fig. 6(b)]. As mentioned previously, the ZBR curve moves to the left, but the single-mode curves are also changed. The single mode quasi-TM curve is again the one that will limit the boundary between the single-mode and multimode behavior, but the stress effect will shift the quasi-TE and quasi-TM curve down. Now, we have very limited locus where both $\mathrm{SMC}$ and ZBC can be matched.

Fig. 7 shows the SM and ZBR curves in the case when a thick oxide layer is deposited onto the waveguide. Fig. 7(a) represents
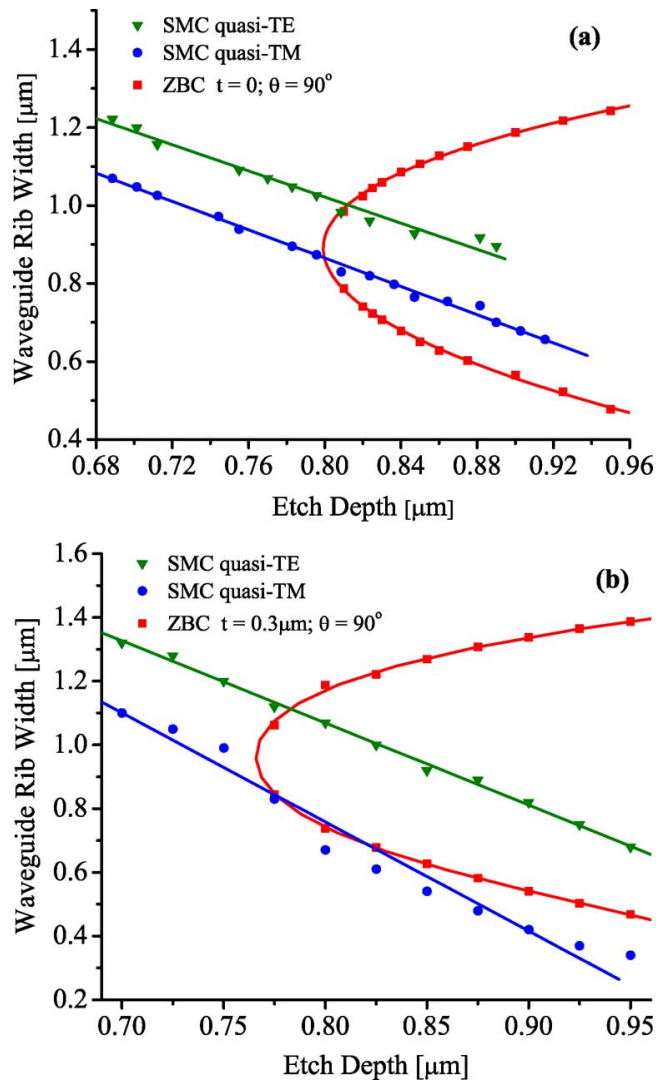

Fig. 6. Single-mode and zero birefringence condition as a function of waveguide rib width and etch depth for top oxide thickness of (a) $t=0$ and (b) $t=$ $0.3 \mu \mathrm{m}\left(\theta=90^{\circ}\right)$.

the waveguide with vertical sidewalls $\left(\theta=90^{\circ}\right)$, while Fig. 7(b) is for the waveguide with slanted sidewalls $\left(\theta=80^{\circ}\right)$. In both figures we present only the quasi-TM mode curves because they define conditions for single-mode behavior. Two cases were analyzed. One is for the isotropic refractive index of material, while the other takes into account the stress-induced effects that produce anisotropic refractive index distribution in the material.

There are three important things that can be seen in Fig. 7(a). First, there is a big difference between the ZBR curves. As it can be seen, the stress-induced birefringence must be taken into account. The ZBR curves moves to the left with respect to the ZBR curve that was calculated in the isotropic case. Hence, there are more choices for waveguide dimensions that allow polarization independence. Second, the single-mode calculation is very important. If the stress effects are taken into account, the curve is slightly different from the one that represents the isotropic case. Here, stress influence is higher for smaller values of etch depth, while for deeper etch depths we obtained similar results as in the isotropic case. Third, the most important thing is that we found the locus where both SM and ZBR conditions are fulfilled. In the isotropic case, the ZBR and SM curves are not close to each other. If we consider the ZBR curve calculated by using stress-induced effects and quasi-TM curve calculated in the isotropic case, we find that the curves are much closer, but again both SM and ZBR conditions cannot be satisfied. Only when the SM and ZBR curves are calculated for the anisotropic 

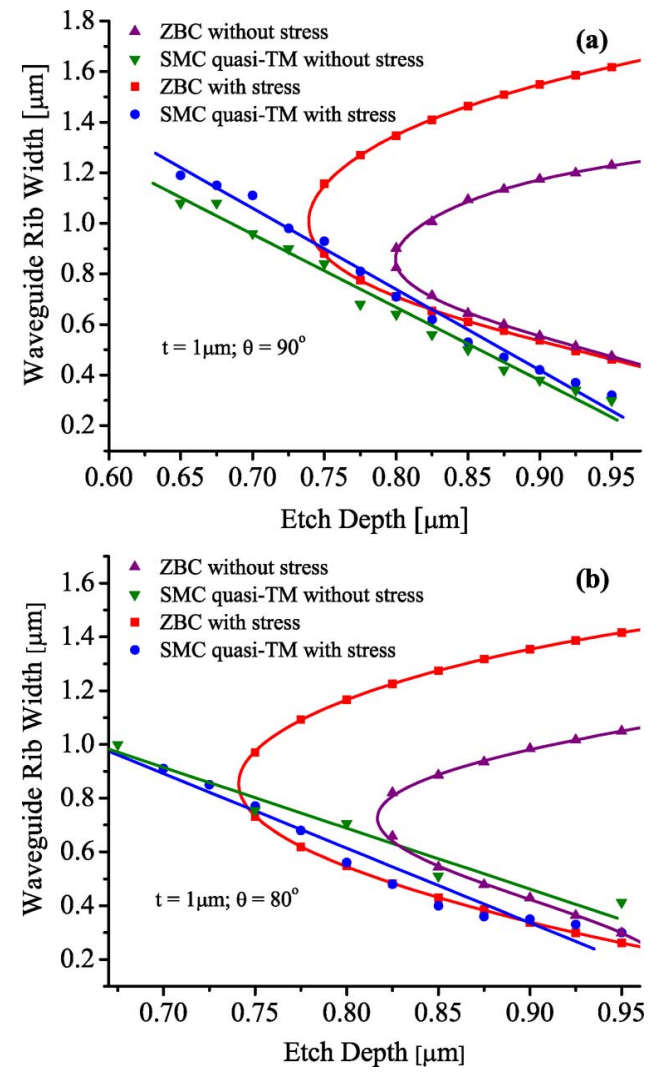

Fig. 7. Single-mode and zero birefringence condition as a function of waveguide rib width and etch depth for top oxide thickness and sidewall angle of (a) $t=1 \mu \mathrm{m}, \theta=90^{\circ}$ and (b) $t=1 \mu \mathrm{m}, \theta=80^{\circ}$.

distribution of the refractive index can both conditions be satisfied. This again shows that the stress engineering needs to be taken into account for SMC and ZBC. In many ways, this is fortuitous result, as it means the stress allows us to fabricate small waveguides that satisfy both the SM and ZBR conditions.

Fig. 7(b) represents a similar situation as Fig. 7(a), but for a waveguide with the slanted sidewalls of $80^{\circ}$ [17], [33]. The ZBR and SM curves are again different. The sidewall angle influences the position of SM curves such that the curve that is calculated using anisotropic distribution of the refractive index is slightly below the curve calculated in the isotropic case, which is different from the situation in Fig. 7(a). In the isotropic case, a relatively short locus that satisfies both $\mathrm{SMC}$ and $\mathrm{ZBC}$ is found, while in the anisotropic case, the desired locus is larger. It is also worth noting that the two SM curves are closer to each other for deeper ribs when $\theta=90^{\circ}$ while the opposite is the case when $\theta=80^{\circ}$. When the sidewall angle $\theta$ decreases, for a certain top rib width $W$ bottom rib width increases. Also, if the waveguide etch depth $D$ increases, for constant $\theta$ and $W$, bottom rib width will increase. In both cases the effective waveguide width increases and consequently the field confinement in the rib. For vertical sidewalls and ribs with large values of $D$, the mode will reside mainly in the slab, and therefore, the influence of the oxide is not significant. Hence, the two SM curves in Fig. 7(a) are similar for deeper ribs. If the sidewalls are slanted, then the difference between the top rib width and the bottom (and also effective) rib width increases with an increase in etch depth. Therefore, the influence of the oxide stress increases and hence the difference between the two SM curves in Fig. 7(b) increases for larger $D$.

\section{CONCLUSION}

In this paper, the design rules that satisfy both single-mode and polarization-independent operation for relatively small cross-sectional SOI rib waveguides have been presented. We have examined waveguides with different sidewall angle, top oxide cover thickness, variable waveguide rib width, and etch depth. The stress effects induced by the upper oxide were discussed and compared with the isotropic case. When the top oxide increases in thickness, the single-mode/multimode boundary is shifted down to lower values of rib widths, compared to the case with an air cladding thickness. The zero birefringence curves moves to smaller values of etch depth and "saturate" for larger values of top oxide cover $(t>0.8 \mu \mathrm{m})$. The ZBR surface is also given to provide the wide range of waveguide dimensions that satisfy the ZBC. It has been shown that by stress engineering, ZBR can be achieved for a waveguide that has otherwise relatively large birefringence. In order to achieve both single-mode and polarization-independent condition, a careful design must be carried out using stress engineering tools, together with the waveguide geometry.

\section{REFERENCES}

[1] R. A. Soref, S. J. Emelett, and W. R. Buchwald, "Silicon waveguided components for the long-wave infrared region," J. Opt. A, Pure Appl. Opt., vol. 8, no. 10, pp. 840-848, Jul. 2006.

[2] C. Gunn, "CMOS photonics: SOI learns a new trick," in Proc. IEEE Int. SOI Conf., New York, Oct. 2005, pp. 7-13.

[3] H. Rong, R. Jones, A. Liu, O. Cohen, D. Hak, A. Fang, and M. Paniccia, "A continuous-wave Raman silicon laser," Nature, vol. 433, pp. 725-728, Feb. 2005.

[4] A. Liu, L. Liao, D. Rubin, H. Nguyen, B. Ciftcioglu, Y. Chetrit, N. Izhaku, and M. Paniccia, "High-speed optical modulation based on carrier depletion in a silicon waveguide," Opt. Exp., vol. 15, no. 2, pp. 660-668, Jan. 2007.

[5] P. Dumon, W. Bogaerts, D. Van Thourhout, D. Taillaert, R. Baets, J. Wouters, S. Beckx, and P. Jaenen, "Compact wavelength router based on a silicon-on-insulator arrayed waveguide grating pigtailed to a fiber array," Opt. Exp., vol. 14, no. 2, pp. 664-669, Jan. 2006.

[6] Y. Nasu, M. Kohtoku, and Y. Hibino, "Low-loss waveguides written with a femtosecond laser for flexible interconnection in a planar lightwave circuit," Opt. Lett., vol. 30, no. 7, pp. 723-725, Apr. 2005.

[7] P. Dumon, W. Bogaerts, V. Wiaux, J. Wouters, S. Beckx, J. Van Campenhout, D. Taillaert, B. Luyssaert, P. Bienstman, D. Van Thourhout, and R. Baets, "Low-loss SOI photonic wires and ring resonators fabricated with deep UV lithography," IEEE Photon. Technol. Lett., vol. 16, no. 5, pp. 1328-1330, May 2004.

[8] T. K. Liang, L. R. Nunes, T. Sakamoto, K. Sasagawa, T. Kawanishi, M. Tsuchiya, G. R. A. Priem, D. V. Thourhout, P. Dumon, R. Baets, and H. K. Tsang, "Ultrafast all-optical switching by cross-absorption modulationin silicon wire waveguides," Opt. Exp., vol. 13, no. 19, pp. 7298-7303, Sep. 2005.

[9] R. A. Soref, J. Schmidtchen, and K. Petermann, "Large single-mode rib waveguides in $\mathrm{Ge}-\mathrm{Si}$ and $\mathrm{Si}-\mathrm{on}-\mathrm{SiO}_{2}$," IEEE J. Quantum Electron., vol. 27, no. 8, pp. 1971-1974, Aug. 1991.

[10] S. P. Pogossian, L. Vescan, and A. Vonsovici, "The single-mode condition for semiconductor rib waveguides with large cross section," $J$. Lightw. Technol., vol. 16, no. 10, pp. 1851-1853, Oct. 1998.

[11] O. Powell, "Single-mode condition for silicon rib waveguides," $J$. Lightw. Technol., vol. 20, no. 10, pp. 1851-1855, Oct. 2002.

[12] L. Vivien, S. Laval, B. Dumont, S. Lardenois, A. Koster, and E. Cassan, "Polarization-independent single-mode rib waveguides on silicon-on-insulator for telecommunication wavelengths," Opt. Commun., vol. 210, no. 1-2, pp. 43-49, Sep. 2002. 
[13] J. Lousteau, D. Furniss, A. Seddon, T. M. Benson, A. Vuković, and P. Sewell, "The single mode condition for silicon-on-insulator optical rib waveguides with large cross section," J. Lightw. Technol., vol. 22, no. 8, pp. 1923-1929, Aug. 2004.

[14] S. P. Chan, C. E. Png, S. T. Lim, V. M. N. Passaro, and G. T. Reed, "Single mode and polarization independent SOI waveguides with small cross section," J. Lightw. Technol., vol. 23, no. 6, pp. 1573-1582, Jun. 2005.

[15] G. Z. Mashanovich, M. Milosevic, P. Matavulj, S. Stankovic, B. Timotijevic, P. Y. Yang, E. J. Teo, M. B. H. Breese, A. A. Bettiol, and G. T. Reed, "Silicon photonic waveguides," Semicon. Scien. Technol., to be published.

[16] W. N. Ye, D.-X. Xu, S. Janz, P. Cheben, M.-J. Picard, B. Lamontagne, and N. G. Tarr, "Birefringence control using stress engineering in silicon-on-insulator (SOI) waveguides," J. Lightw. Technol., vol. 23, no. 3, pp. 1308-1318, Mar. 2005.

[17] M. M. Milošević, P. S. Matavulj, and G. Z. Mashanovich, "Stress-induced characteristics of silicon-on-insulator rib waveguides," in Proc. 15th Telecommun. Forum TELFOR, Belgrade, Serbia, Nov. 2007, pp. 401-404.

[18] S. Stanković, M. Milošević, B. Timotijević, P. Y. Yang, E. J. Teo, J. Crnjanski, P. Matavulj, and G. Z. Mashanovich, "Silicon photonic waveguides for near- and mid- infrared regions," Acta Phys. Polonica A, vol. 112, no. 5, pp. 1019-1024, Nov. 2007.

[19] W. R. Headley, G. T. Reed, M. Pannicia, A. Liu, and S. Howe, "Polarization-independent optical racetrack resonators using rib waveguides in silicon-on-insulator," Appl. Phy. Lett., vol. 85, no. 23, pp. 5523-5525, Dec. 2004.

[20] B. D. Timotijevic, F. Y. Gardes, W. R. Headley, G. T. Reed, M. J. Paniccia, O. Cohen, D. Hak, and G. Z. Masanovic, "Multi-stage racetrack resonator filters in silicon-on-insulator," J. Opt. A, Pure Appl. Opt., vol. 8, no. 7, pp. S473-S476, Jul. 2006.

[21] K. Kawano and T. Kitoh, Introduction to Optical Waveguide Analysis: Solving Maxwell's Equation and the Schrödinger Equation. New York: Wiley, 2001.

[22] R. Scarmozzino, A. Gopinath, R. Pregla, and S. Helfert, "Numerical techniques for modeling guided-wave photonic devices," IEEE J. Sel. Topics Quantum Electron., vol. 6, no. 1, pp. 150-162, Jan./Feb. 2000.

[23] FEMLAB COMSOL, Inc., Burlington, MA [Online]. Available: www. comsol.com

[24] Beamprop Rsoft Design Group, Inc., Ossining, NY [Online]. Available: www.rsoftdesign.com

[25] M. Huang, "Stress effects on the performance of optical waveguides," Int. J. Sol. Structures, vol. 40, no. 7, pp. 1615-1632, Apr. 2003.

[26] K. Okamoto, T. Hosaka, and T. Edahiro, "Stress analysis of optical fibers by a finite element method," IEEE J. Quantum Electron., vol. QE-17, no. 10, pp. 2123-2129, Oct. 1981.

[27] M. Okuno, A. Sugita, K. Jinguji, and M. Kawachi, "Birefringence control of silica waveguides on $\mathrm{Si}$ and its application to a polarization-beam splitter/swich," J. Lightw. Technol., vol. 12, no. 4, pp. 625-633, Apr. 1994.

[28] K. Saitoh, M. Koshiba, and Y. Tsuji, "Stress analysis method for elastically anisotropic material based optical waveguides and its application to stess-induced optical waveguides," J. Lightw. Technol., vol. 17, no. 2, pp. 255-259, Feb. 1999.

[29] D.-X. Xu, P. Cheben, D. Dalacu, A. Delage, S. Janz, B. Lamontagne, M.-J. Picard, and W. N. Ye, "Eliminating the birefringence in silicon-on-insulator ridge waveguides by use of cladding stress," Opt. Lett., vol. 29, no. 20, pp. 2384-2386, Oct. 2004.

[30] W. N. Ye, D. X. Xu, S. Janz, P. Cheben, A. Delage, M.-J. Picard, B. Lamontagne, and G. T. Tarr, "Stress-induced birefringence in silicon-on-insulator (SOI) waveguides," in Proc. SPIE-Int. Soc. Opt. Eng., Jul. 2004, vol. 5357, pp. 57-66.

[31] S. Timoshenko and J. N. Goodier, Theory of Elasticity, 2nd ed. New York: McGraw-Hill, 1951.

[32] L. D. Landau and E. M. Lifshitz, Theory of Elasticity, 2nd ed. Oxford, U.K.: Pergamon, 1970.

[33] B. D. Timotijevic, "Auto-regressive optical filters in silicon-on-insulator waveguides," Ph.D. dissertation, Adv. Technol. Inst., Univ. Surrey, Guildford, U.K., 2007.
Milan M. Milošević was born in Serbia in 1983. He received the Dipl. Ing. degree from the Faculty of Electrical Engineering, University of Belgrade, Belgrade, Serbia, in 2007, where he is currently working towards the Ph.D. degree.

In 2006, he was an internship student in Keihanna Technology Innovation Center, OMRON Corporation, Kyoto, Japan, where he made a software to predict the error range of thin film thickness by using spectroscopic ellipsometer. His research interests encompass integrated optics, quantum optics and nanophotonics. He is currently engaged in the Royal Society U.K. International Joint Project.

Petar S. Matavulj (S'96-M'02) received the B.S., M.S., and Ph.D. degrees in electrical engineering from the University of Belgrade, Serbia, in 1994, 1997, and 2002, respectively.

Currently, he is an Associate Professor of Physical Electronics at Faculty of Electrical Engineering, University of Belgrade, Belgrade, Serbia. From 1994 to 2002, he was an Assistant at the Faculty of Electrical Engineering, University of Belgrade. His research interests are modeling, simulation and characterization of high-speed optoelectronic devices, integrated optics and optical communications and networks.

Dr. Matavulj is a member of the IEEE Lasers and Electro-Optics Society (LEOS), Electron Device Society (EDS), and Communications Society (ComSoc).

Branislav D. Timotijević was born in Serbia in 1978. He received the B.Sc.E.E. degree in optoelectronics from Faculty of Electrical Engineering, Belgrade University, Belgrade, Serbia, in 2002. In April 2004, he received an Overseas Research Scholarship (ORS) Award and sponsorship from the Intel Corporation to pursue Ph.D. studies at University of Surrey, UK, in the area of silicon-based integrated photonics under the supervision of Prof. G.T. Reed.

Currently, he is a Research Fellow at "Vinca" Institute of Nuclear Sciences, Belgrade, Serbia, investigating ion beam modifications of AlTi and AlNTiN multilayers. His current research interests include integrated photonics and light and electron microscopy.

Graham T. Reed received the B.Sc. and Ph.D. degrees from University of Surrey, Surrey, Guildford, U.K., in 1983 and 1987, respectively.

He has been active in research in guided wave optics since approximately 1983, and in May 1989, he joined the University of Surrey, where he formed the Silicon Photonics Research Group. The group has produced a series of leading technical advances in the field worldwide. He was the principal author of the first textbook on silicon photonics and has authored five further book chapters in other publications. He has also contributed to several patents and has published over 200 papers.

Prof. Reed is a member of three international conference committees related to silicon photonics. He is Co-Chair of the Silicon Photonics Symposium that is part of Photonics West, held annually in San Jose, CA, one of the largest annual photonics meetings. He is a Fellow of the Institution of Engineering and Technology (IET).

Goran Z. Mashanovich received the Dipl. Ing. degree and M.Sc. degree in electrical engineering from the Faculty of Electrical Engineering, University of Belgrade, Belgrade, Serbia, in 1995 and 1999, respectively, and the Ph.D. degree from the University of Surrey, Guildford, U.K., in 2005.

After working for five years as a Teaching and Research Assistant at the Faculty of Electrical Engineering, University of Belgrade, he joined the Silicon Photonics Group, Faculty of Engineering and Physical Sciences, University of Surrey, in 2000 . He is currently the group manager. His research interests include silicon photonics and fiber optic sensors. 